\title{
Chemistry of Hydrogen Fluoride in the Interstellar Medium
}

\section{Citation}

Zhu, C., R. Krems, A. Dalgarno, and N. Balakrishnan. 2002. “Chemistry of Hydrogen

Fluoride in the Interstellar Medium." The Astrophysical Journal 577 (2): 795-97. https://

doi.org/10.1086/342240.

\section{Permanent link}

http://nrs.harvard.edu/urn-3:HUL.InstRepos:41397401

\section{Terms of Use}

This article was downloaded from Harvard University's DASH repository, and is made available under the terms and conditions applicable to Other Posted Material, as set forth at http:// nrs.harvard.edu/urn-3:HUL.InstRepos:dash.current.terms-of-use\#LAA

\section{Share Your Story}

The Harvard community has made this article openly available.

Please share how this access benefits you. Submit a story.

Accessibility 


\title{
CHEMISTRY OF HYDROGEN FLUORIDE IN THE INTERSTELLAR MEDIUM
}

\author{
C. Zhu, R. Krems, and A. Dalgarno \\ Harvard-Smithsonian Center for Astrophysics, 60 Garden Street, Cambridge, MA 02138 \\ AND \\ N. BALAKRISHNAN \\ Department of Chemistry, University of Nevada, 4505 Maryland Parkway, Las Vegas, NV 89154 \\ Received 2002 April 15; accepted 2002 June 8
}

\begin{abstract}
A comprehensive account is given of the chemistry of HF in interstellar clouds. Calculations are presented of the rate coefficients for the quenching of $\mathrm{F}\left({ }^{2} P_{1 / 2}\right)$ and for the reaction of $\mathrm{F}\left({ }^{2} P_{3 / 2}\right)$ atoms in collisions with $\mathrm{H}_{2}$. They confirm the conclusions of Neufeld and coworkers that gaseous fluorine exists in interstellar clouds almost entirely in the form of the molecule $\mathrm{HF}$.
\end{abstract}

Subject headings: astrochemistry — ISM: molecules — molecular data — molecular processes

\section{INTRODUCTION}

Hydrogen fluoride has been detected in sunspots (Hall \& Noyes 1969; Wallace \& Livingston 1991; Wallace, Livingston, \& Bernath 1994), in the atmospheres of Venus (Connes et al. 1967), $\alpha$ Ori (Spinrad et al. 1970), and red giants (Jorissen, Smith, \& Lambert 1992), and in the interstellar medium (Neufeld et al. 1997). It was discovered in the interstellar medium through the observation of an absorption line at $121.6973 \mu \mathrm{m}$ attributed to the $J=2-1$ rotational transition of HF (Neufeld et al. 1997). The line was detected in the line of sight toward the far-infrared continuum source Sgr B2. The fractional abundance relative to $\mathrm{H}_{2}$ derived is $3 \times 10^{-10}$. Neufeld et al. (1997) present a brief discussion of the fluorine chemistry, and they conclude that most of the fluorine will be taken up as HF, the small observed abundance of HF then implying that fluoride is substantially depleted onto interstellar grains. Neufeld et al. (1997) based their discussion of the chemistry on the reactions

$$
\mathrm{F}+\mathrm{H}_{2} \rightarrow \mathrm{HF}+\mathrm{H}
$$

and

$$
\mathrm{F}+\mathrm{H}_{2} \mathrm{O} \rightarrow \mathrm{HF}+\mathrm{OH} .
$$

Measurements of the rate coefficients have been carried out at temperatures above 200 K (Wurzberg \& Houston 1980; Heidner et al. 1980; Stevens, Brune, \& Anderson 1989). Neufeld et al. (1997) concluded that only reaction (1) was important in the astrophysical environment, except possibly at very cool temperatures. They extrapolated the rate coefficient $k_{1}$ of reaction (1) to temperatures below $200 \mathrm{~K}$ using the representation

$$
k_{1}=1.4 \times 10^{-10} \exp (-500 / T) \mathrm{cm}^{3} \mathrm{~s}^{-1},
$$

recommended by Atkinson et al. (1992). The chemistry is more complicated because the rate coefficient of reaction (1) depends on the fine-structure population of the fluorine atom and because it may also depend on the rotational state of the hydrogen molecule.

The fine-structure population is determined by a balance between the radiative decay of $\mathrm{F}\left({ }^{2} P_{1 / 2}\right)$ and collisioninduced transitions

$$
\mathrm{F}\left({ }^{2} P_{1 / 2}\right)+\mathrm{H}_{2} \leftrightarrow \mathrm{F}\left({ }^{2} P_{3 / 2}\right)+\mathrm{H}_{2} .
$$

We present here the results of fully quantum-mechanical calculations of the rate coefficients of processes (1) and (4) and discuss the resulting modifications to the chemistry of $\mathrm{HF}$.

\section{FINE-STRUCTURE COLLISIONS}

There have been several theoretical studies of transitions between fine-structure levels of $\mathrm{F}$ induced by collisions with $\mathrm{H}_{2}$ (Rebentrost \& Lester 1976; Fitz \& Kouri 1981; McNutt \& Wyatt 1981; Gilibert \& Baer 1995) with results that show some sensitivity to the interaction potentials. A reliable interaction potential has been calculated by Alexander, Manolopoulos, \& Werner (2000), and we adopt it here. The relevant quantum-mechanical scattering theory has been developed by Baer (1975), Alexander (1993, 1995), Dubernet \& Hutson (1994a, 1994b), and Alexander et al. (2000). Details of the analytical and numerical procedures that we followed to calculate the collision cross sections are given by Alexander et al. (2000) and Krems \& Dalgarno (2002).

It was discovered by Rebentrost \& Lester (1976) that the relaxation of $\mathrm{F}\left({ }^{2} P_{1 / 2}\right)$ colliding with $\mathrm{H}_{2}$ in the lowest rotational level $j=0$ at energies between 35 and $700 \mathrm{~cm}^{-1}$ is dominated by the near-resonance reaction

$$
\begin{aligned}
& \mathrm{F}\left({ }^{2} P_{1 / 2}\right)+\mathrm{H}_{2}(j=0) \rightarrow \\
& \mathrm{F}\left({ }^{2} P_{3 / 2}\right)+\mathrm{H}_{2}(j=2)+49.82 \mathrm{~cm}^{-1},
\end{aligned}
$$

in which $\mathrm{H}_{2}$ is excited from the $j=0$ to the $j=2$ level. Our calculations confirmed that process (5) occurs much more rapidly than the reaction

$$
\begin{aligned}
\mathrm{F}\left({ }^{2} P_{1 / 2}\right)+\mathrm{H}_{2}(j=0) \rightarrow & \mathrm{F} \\
& \left(P_{3 / 2}\right) \\
& +\mathrm{H}_{2}(j=0)+404 \mathrm{~cm}^{-1},
\end{aligned}
$$

in which $\mathrm{H}_{2}$ does not undergo any change in its rotational level. The near-resonance occurs only for $j=0$, so that quenching of $\mathrm{F}\left({ }^{2} P_{1 / 2}\right)$ in collisions with $\mathrm{H}_{2}$ in higher rotational levels $j \geq 1$,

$$
\mathrm{F}\left({ }^{2} P_{1 / 2}\right)+\mathrm{H}_{2}(j \geq 1) \rightarrow \mathrm{F}\left({ }^{2} P_{3 / 2}\right)+\mathrm{H}_{2},
$$

is also relatively slow. 
TABLE

Rate Coefficients for Relaxation of F $\left({ }^{2} P_{1 / 2}\right)$

\begin{tabular}{cccc}
\hline \hline $\begin{array}{c}T \\
(\mathrm{~K})\end{array}$ & $\begin{array}{c}\text { Reaction (5) } \\
\left(\mathrm{cm}^{3} \mathrm{~s}^{-1}\right)\end{array}$ & $\begin{array}{c}\text { Reaction }(6) \\
\left(\mathrm{cm}^{3} \mathrm{~s}^{-1}\right)\end{array}$ & $\begin{array}{c}\text { Reaction }(7) \\
\left(\mathrm{cm}^{3} \mathrm{~s}^{-1}\right)\end{array}$ \\
\hline $50 \ldots \ldots \ldots$ & $0.226 \times 10^{-9}$ & $0.249 \times 10^{-11}$ & $0.546 \times 10^{-11}$ \\
$100 \ldots \ldots$ & $0.288 \times 10^{-9}$ & $0.528 \times 10^{-11}$ & $0.915 \times 10^{-10}$ \\
$150 \ldots \ldots$ & $0.315 \times 10^{-9}$ & $0.876 \times 10^{-11}$ & $0.132 \times 10^{-10}$ \\
$200 \ldots \ldots$ & $0.329 \times 10^{-9}$ & $0.127 \times 10^{-10}$ & $0.177 \times 10^{-10}$ \\
$250 \ldots \ldots$ & $0.338 \times 10^{-9}$ & $0.169 \times 10^{-10}$ & $0.226 \times 10^{-10}$ \\
$300 \ldots \ldots$ & $0.342 \times 10^{-9}$ & $0.213 \times 10^{-10}$ & $0.278 \times 10^{-10}$ \\
$350 \ldots \ldots$ & $0.345 \times 10^{-9}$ & $0.260 \times 10^{-10}$ & $0.333 \times 10^{-10}$ \\
$400 \ldots \ldots$ & $0.346 \times 10^{-9}$ & $0.309 \times 10^{-10}$ & $0.389 \times 10^{-10}$ \\
$450 \ldots \ldots$ & $0.346 \times 10^{-9}$ & $0.358 \times 10^{-10}$ & $0.446 \times 10^{-10}$ \\
$500 \ldots \ldots$ & $0.346 \times 10^{-9}$ & $0.409 \times 10^{-10}$ & $0.503 \times 10^{-10}$ \\
$550 \ldots \ldots$ & $0.345 \times 10^{-9}$ & $0.459 \times 10^{-10}$ & $0.560 \times 10^{-10}$ \\
$600 \ldots \ldots$ & $0.344 \times 10^{-9}$ & $0.510 \times 10^{-10}$ & $0.615 \times 10^{-10}$ \\
$650 \ldots \ldots$ & $0.342 \times 10^{-9}$ & $0.560 \times 10^{-10}$ & $0.670 \times 10^{-10}$ \\
$700 \ldots \ldots$ & $0.341 \times 10^{-9}$ & $0.610 \times 10^{-10}$ & $0.720 \times 10^{-10}$ \\
\hline
\end{tabular}

Notes. - For reactions $(5)$ and (6) initial $j=0$, and for reaction (7) initial $j=1$.

In Table 1 we present the rate coefficients for processes (5) and (6) for $j=0$ and process (7) for $j=1$. The rates of the reverse excitation processes can be obtained by detailed balance. The calculations of the rate coefficients for reaction (5) are fully converged and the uncertainty of rate coefficients for reactions (6) and (7) due to numerical integration is within $10 \%$. For the reverse of process (5) to be effective requires a significant population of $j=2$ levels of $\mathrm{H}_{2}$. In practice in the interstellar environment, given our calculated rate coefficients and the short radiative lifetime $1.10 \times 10^{3} \mathrm{~s}$ of $\mathrm{F}\left({ }^{2} P_{1 / 2}\right)$ (Cheng, Kim, \& Desclaux 1979), all the $\mathrm{F}$ atoms can be taken to reside in the ground ${ }^{2} P_{3 / 2}$ state.

\section{THE REACTION OF $\left.\mathrm{F}^{2} P_{3 / 2}\right)$ WITH $\mathrm{H}_{2}$}

Reaction (1) is a benchmark case that has been the subject of extensive theoretical and experimental study (Rosenman et al. 1996; Castillo et al. 1996; Russell \& Manolopolous 1996; Chapman et al. 1998; Castillo et al. 1998; Manolopolous 1997; Takayanagi \& Kurosaki 1998; Alexander, Werner, \& Manolopoulos 1998; Baer et al. 1999; Ma, Cai, \& Deng 1999; Balakrishnan \& Dalgarno 2001). Alexander et al. (2000) have shown that the reaction dynamics is represented satisfactorily by scattering on the lowest potential energy surface of Stark \& Werner (1997). Our calculations of the rate coefficients are based on this assumption.

We have used the $\mathrm{ABC}$ quantum reactive scattering program of Skouteris, Castillo, \& Manolopoulos (2000), which couples channels with a given total angular momentum quantum number $J$. Total angular momentum quantum numbers up to $J=20$ were required to secure convergence for collision energies up to $0.2 \mathrm{eV}$. Many product rotational states are populated in the reaction. The individual partial cross sections show the presence of resonances in the product channels but the rate coefficients are smooth functions of temperature. We report in Table 2 the rate coefficients for the reactions

$$
\mathrm{F}\left({ }^{2} P_{3 / 2}\right)+\mathrm{H}_{2}(j=0) \rightarrow \mathrm{HF}+\mathrm{H}
$$

TABLE 2

Rate CoefFicients For Reactions (8) AND (9) OF F $\left({ }^{2} P_{3 / 2}\right)$

\begin{tabular}{ccc}
\hline \hline $\begin{array}{c}T \\
(\mathrm{~K})\end{array}$ & $\begin{array}{c}\text { Reaction }(8) \\
\left(10^{-11} \mathrm{~cm}^{3} \mathrm{~s}^{-1}\right)\end{array}$ & $\begin{array}{c}\text { Reaction }(9) \\
\left(10^{-11} \mathrm{~cm}^{3} \mathrm{~s}^{-1}\right)\end{array}$ \\
\hline $50 \ldots \ldots .$. & 0.312 & 0.184 \\
$100 \ldots \ldots$. & 0.791 & 0.476 \\
$150 \ldots \ldots$. & 1.32 & 0.974 \\
$200 \ldots \ldots$. & 1.92 & 1.60 \\
$250 \ldots \ldots$. & 2.54 & 2.29 \\
$300 \ldots \ldots$. & 3.16 & 2.98 \\
$350 \ldots \ldots$. & 3.75 & 3.63 \\
$400 \ldots \ldots$. & 4.28 & 4.21 \\
$450 \ldots \ldots$. & 4.74 & 4.72 \\
$500 \ldots \ldots$. & 5.13 & 5.14 \\
\hline
\end{tabular}

Notes.-Reaction $(8): \mathrm{H}_{2}(j=0)$; reaction (9): $\mathrm{H}_{2}(j=1)$.

and

$$
\mathrm{F}\left({ }^{2} P_{3 / 2}\right)+\mathrm{H}_{2}(j=1) \rightarrow \mathrm{HF}+\mathrm{H}
$$

at temperatures between 50 and $500 \mathrm{~K}$.

The experimental values (Wurzberg \& Houston 1980; Heidner et al. 1980; Stevens et al. 1989) refer presumably to measurements with thermal populations of fine-structure levels and rotational populations. We find little dependence on $j$ until temperatures fall below $250 \mathrm{~K}$. Alexander et al. (1998) have found that the reactivity of $\mathrm{F}\left({ }^{2} P_{1 / 2}\right)$ is less than $10 \%$ of that of $\left.\mathrm{F}^{2} P_{3 / 2}\right)$ so that in order to compare with the measured values, our calculated rate coefficients for $\mathrm{F}\left({ }^{2} P_{3 / 2}\right)$ should be multiplied by a factor of $2 /\left(2+e^{-\Delta / k_{\mathrm{B}} T}\right)$, where $\Delta=50.1 \mathrm{meV}$ is the ${ }^{2} P_{1 / 2}{ }^{2} P_{3 / 2}$ splitting of the $\mathrm{F}$ atom, $k_{\mathrm{B}}$ is Boltzmann's constant, and $T$ is the temperature. A comparison that includes this factor is given in Figure 1. The agreement between the theoretical and experimental data is good. In Figure 1, we also include some previous theoretical calcu-

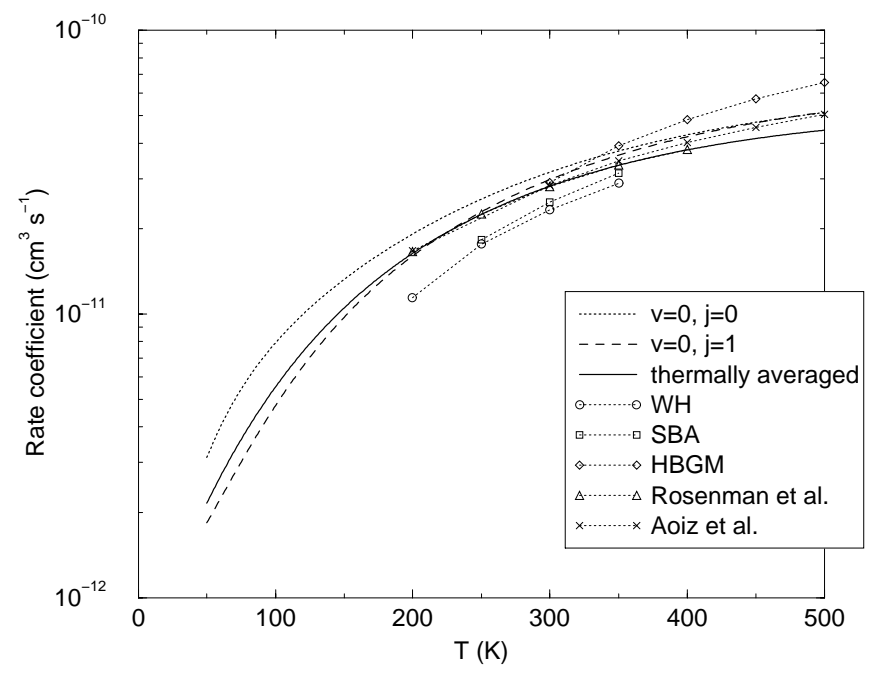

FIG. 1.-Rate coefficients for the $\mathrm{F}+\mathrm{H}_{2}$ reaction: solid curve, thermally averaged rate coefficients; dotted curve, $\mathrm{F}\left({ }^{2} P_{3 / 2}\right)+\mathrm{H}_{2}(v=0, j=0)$ reaction; dashed curve, $\mathrm{F}\left({ }^{2} P_{3 / 2}\right)+\mathrm{H}_{2}(v=0, j=1)$ reaction; circles, squares, and diamonds are the experimental data of Wurzberg \& Houston (1980), Stevens et al. (1989), and Heidner et al. (1980), respectively. Triangles are the calculations by Rosenman et al. (1996), and crosses are the values by Aoiz et al. (1999). 
TABLE 3

Coefficients for the Analytical Approximations (Eq. [10]) of the Temperature Dependence of the Rate Coefficients for the Reaction of Fluorine with $\mathrm{H}_{2}(j)$

\begin{tabular}{cccccc}
\hline \hline$i$ & 0 & 1 & 2 & 3 & 4 \\
\hline$j=0 \ldots \ldots$ & -10.9967 & -289.052 & 22486.0 & $-9.57330 \times 10^{5}$ & $1.58973 \times 10^{7}$ \\
$j=1 \ldots \ldots$. & -10.9829 & -278.212 & 12884.0 & $-2.09014 \times 10^{5}$ & $-6.7910 \times 10^{5}$ \\
\hline
\end{tabular}

lations. The results of approximate coupled-states calculations by Roseman et al. (1996) agree very well with our calculations in the temperature range of $200-400 \mathrm{~K}$, as do those of Aoiz, Banares, \& Castillo (1999) at temperatures below $350 \mathrm{~K}$, obtained with the adiabatic rotation approximation. The adiabatic rotation approximation treats the rotational motion of the triatomic system adiabatically by neglecting the Coriolis coupling. At higher temperatures the values of Aoiz et al. (1999) are larger than ours, indicating a loss of accuracy in their approximation at high temperatures.

At $100 \mathrm{~K}$, the predicted rate coefficient is 11.8 times that given by equation (3) and adopted by Neufeld et al. (1997). The theoretical results can be represented as a function of temperature by

$$
\ln k(T)=\sum_{i=0}^{4} \frac{a_{i}}{T^{i}}
$$

The fitting coefficients in equation (10) for the reactions of $\left.\mathrm{F}^{2} P_{3 / 2}\right)$ with $\mathrm{H}_{2}(j=0)$ and $\mathrm{H}_{2}(j=1)$ are given in Table 3 .

\section{CONCLUSIONS}

Because in the interstellar medium the $\mathrm{F}$ atom resides in the $\left({ }^{2} P_{3 / 2}\right)$ state and because it is the $\left({ }^{2} P_{3 / 2}\right)$ state that is highly reactive in collisions with $\mathrm{H}_{2}$, the conclusions of Neufeld et al. (1997) that F is converted almost entirely to HF is strengthened by our more comprehensive discussion of the chemistry.

This work was supported by the Division of Astronomy of the National Science Foundation and the University of Nevada at Las Vegas Research Office through a New Investigator Award to N. B. We are grateful to David Neufeld for his comments on an earlier draft.
Alexander, M. H. 1993, J. Chem. Phys., 99, 6014

Alexander, M. H., Manolopoulos, D. E., \& Werner, H.-J. 2000, J. Chem. Phys. 113, 11084

Alexander, M. H., Werner, H. J., \& Manolopoulos, D. E. 1998, J. Chem. Phys., 109, 5710

Alexander, M. H., \& Yang, M. 1995, J. Chem. Phys., 103, 7956

Aoiz, F. J., Banares, L., \& Castillo, J. F. 1999, J. Chem. Phys., 111, 4013

Atkinson, R., Baulch, D. L., Cox, R. A., Hampson, R. F., Jr., Kerr, J. A., \& Troe, J. 1992, J. Phys. Chem. Ref. Data, 21, 1125

Baer, M. 1975, Chem. Phys. Lett., 35, 112

Baer, M., Faubel, M., Martinez-Haya, B., Rusin, L., Tappe, U., \& Toennies, J. P. 1999, J. Chem. Phys., 110, 10231

Balakrishnan, N., \& Dalgarno, A. 2001, Chem. Phys. Lett., 341, 652 (erratum, 351, 159 [2002])

Castillo, J. F., Hartke, B., Werner, H. J., Aoiz, F. J., Banares, L., \& Martinezhaya, B. 1998, J. Chem. Phys., 109, 7224

Castillo, J. F., Manolopoulos, D. E., Stark, K., \& Werner, H. J. 1996, J. Chem. Phys., 104, 6531

Chapman, W. B., Blackmon, B. W., Nizkorodov, S., \& Nesbitt, D. J. 1998 , J. Chem. Phys., 109, 9306

Cheng, K. T., Kim, Y.-K., \& Desclaux, J. P. 1979, A. Data Nucl. Data Tables, 24, 111

Connes, P., Connes, J., Benedict, W. S., \& Kaplan, L. D. 1967, ApJ, 147, 1230

Dubernet, M.-L., \& Hutson, J. 1994a, J. Chem. Phys., 101, 1939

. 1994b, J. Phys. Chem., 98, 5844

Fitz, D. E., \& Kouri, D. J. 1981, J. Chem. Phys., 74, 3933

Gilibert, M., \& Baer, M. 1995, J. Phys. Chem., 99, 15748

Hall, D. N. B., \& Noyes, R. W. 1969, Astrophys. Lett., 4, 143

\section{REFERENCES}

Heidner III, R. F., Bott, J. F., Gardner, C. E., \& Meizer, J. E. 1980, J. Chem. Phys., 72, 4815

Jorissen, A., Smith, V. V., \& Lambert, D. L. 1992, A\&A, 261, 164

Krems, R. V., \& Dalgarno, A. 2002, J. Chem. Phys., 117, 118

Ma, W.-Y., Cai, Z.-T., \& Deng, C.-H. 1999, Chem. Phys. Lett., 304, 121

Manolopoulos, D. E. 1997, J. Chem. Soc. Faraday Trans., 93, 673

McNutt, J. F., \& Wyatt, R. E. 1981, Chem. Phys., 58, 423

Neufeld, D. A., Zmuidzinas, J., Schilke, P., \& Phillips, T. G. 1997, ApJ, 488, L141

Rebentrost, F., \& Lester, W. A. 1976, J. Chem. Phys., 64, 3879

Rosenman, E., Hochman-Kowal, S., Persky, A., \& Baer, M. 1996, Chem. Phys. Lett., 257, 421

Russell, C. L., \& Manolopoulos, D. E. 1996, Chem. Phys. Lett., 256, 465

Skouteris, D., Castillo, J. F., \& Manolopoulos, D. E. 2000, Comput. Phys. Commun., 133, 128

Spinrad, H., Kaplan, L. D., Connes, P., Connes, J., Kunde, V. G., \& Maillard, J. P. 1970, in Proc. Conf. Late Type Stars, ed. G. W. Lockwood $\&$ H. M. Dyck (Tucson: KPNO), 59

Stark, K., \& Werner, H.-J. 1996, J. Chem. Phys., 104, 6515

Stevens, P.S., Brune, W. H., \& Anderson, J. G. 1989, J. Phys. Chem., 93 , 4068

Takayanagi, T., \& Kurosaki, Y. 1998, Chem. Phys. Lett., 286, 35

Wallace, L., \& Livingston, W. 1991, J. Geophys. Res., 96, 15513

Wallace, L., Livingston, W., \& Bernath, P. 1994, Atlas of Sunspot Spectrum from 470 to $1233 \mathrm{~cm}^{-1}$ (8.1 to 21 Micrometer) and the Photospheric Spectrum from 460 to $630 \mathrm{~cm}^{-1}$ (16 to 22 Micrometer) (NSO Tech. Rep. 94-001; Tucson: Natl. Solar Obs. (NOAO)

Wurzberg, E., \& Houston, P. L. 1980, J. Chem. Phys., 72, 4811 


\section{ERRATUM: “CHEMISTRY OF HYDROGEN FLUORIDE IN THE INTERSTELLAR MEDIUM” (2002, ApJ, 577, 795)}

C. Zhu ${ }^{1}$, R. Krems $^{1}$, A. Dalgarno ${ }^{1}$, and N. Balakrishnan ${ }^{2}$

${ }^{1}$ Harvard-Smithsonian Center for Astrophysics, 60 Garden Street, Cambridge, MA 02138, USA

${ }^{2}$ Department of Chemistry, University of Nevada Las Vegas, 4505 Maryland Parkway, Las Vegas, NV 89154, USA

The fitting coefficients reported in Table 3 of the original article are incorrect. The corrected values are given below and are valid in the temperature range of 50-500 K. We thank Millard Alexander for bringing this to our attention.

Table 3

Coefficients for the Analytical Approximations (Equation (10)) of the Temperature Dependence of the Rate Coefficients for the Reaction of Fluorine with $\mathrm{H}_{2}(j)$

\begin{tabular}{ccccrr}
\hline \hline$i$ & 0 & 1 & 2 & 3 & 4 \\
\hline$j=0$ & -22.7604 & -523.612 & 32220.0 & $-8.04202 \times 10^{5}$ & $1.83287 \times 10^{6}$ \\
$j=1$ & -22.7603 & -481.476 & 8187.27 & $1.11067 \times 10^{6}$ & $-4.24469 \times 10^{7}$ \\
\hline
\end{tabular}

